\title{
Primary vaginal adenocarcinoma of intestinal type or occult metastatic colon cancer: a diagnostic dilemma from a vaginal skin tag
}

This article was published in the following Dove Medical Press journal: International Journal of Women's Health

\author{
Anna Louise Russell' \\ Ben Haagsma ${ }^{2}$ \\ Thumuluru Kavitha \\ Madhuri ${ }^{3,4}$ \\ 'Department of Gynae-Oncology, \\ Royal Surrey County Hospital NHS \\ Foundation Trust, Guildford, UK; \\ ${ }^{2}$ Department of Histopathology, \\ Royal Surrey County Hospital NHS \\ Foundation Trust, Guildford, UK; \\ ${ }^{3}$ Department of Gynaecological \\ Oncology, Royal Surrey County \\ Hospital NHS Foundation Trust, \\ Guildford, UK; ${ }^{4}$ Department of \\ Experimental Medicine, Faculty of \\ Health \& Medical Sciences, University \\ of Surrey, Guildford, UK
}

\begin{abstract}
The presentation of a new vaginal lesion could represent a variety of diagnoses from benign warts to more sinister primary malignancies. Rarely, a new lesion could represent a metastatic deposit from a malignancy elsewhere in the body. Colonic carcinomas are the third most common malignancy, frequently metastasising to the liver and lung. There have been a small number of cases in the literature reporting vaginal metastases from colonic carcinoma and this is usually indicative of advanced disseminated disease. We present an interesting case of a 65 -yearold female with a strong family history of bowel cancer who originally presented with a vaginal skin tag that was biopsied and found to be a moderately differentiated adenocarcinoma. The immunohistochemistry profile was cytokeratin (CK) 20 positive/CK 7 negative, highly suggestive of a bowel cancer primary. However, subsequent extensive radiological and endoscopic investigations failed to identify a colonic primary tumor. The vaginal lesion was successfully excised, and no systemic treatments were warranted. To date, no primary cancer has been identified; the patient remains asymptomatic with no clinical signs of disease recurrence 5 years following her initial diagnosis. This case represents a diagnostic dilemma between two very rare diagnoses of either a vaginal metastasis from an occult colonic primary tumor or a primary vaginal adenocarcinoma of endometrioid morphology demonstrating intestinal immunophenotype. Organizing colonic screening is recommended in view of the high risk of colonic adenocarcinoma.
\end{abstract}

Keywords: skin tag, vaginal cancer, metastases, occult malignancy, colon cancer, villiform, intestinal villiform, endometrioid

\section{Introduction}

The initial presentation of a new vaginal lesion may represent a number of differential diagnoses. It could be a simple skin tag or benign wart, yet rarely may mask a more sinister diagnosis. An apparently innocent skin tag may be the first presentation of a vaginal malignancy or rarer still a metastases from a malignancy elsewhere in the body, as shown in our unusual case of colorectal adenocarcinoma masquerading as a vaginal skin tag.

\section{Case summary}

A 65-year-old female presented to her general practitioner with post-menopausal bleeding. On vaginal examination, there was a polyp-like lesion at the vagina introitus that appeared to be the source of the bleeding. Systemic examination, past medical history, and gynecological history were otherwise unremarkable. Her family history included her father who died aged 60 from metastatic colonic cancer and her mother, aged 75 , who had an excision of locally advanced colonic carcinoma. \footnotetext{
Madhuri

Department of Gynae-Oncology, Royal Surrey County Hospital NHS Foundation Trust, LEVEL B GOPD, Guildford, GU2 7XX, UK

Email t.madhuri@surrey.ac.uk
}

Correspondence: Thumuluru Kavitha 
Following referral to the gynecology department, vaginal examination revealed a painless skin tag at the introitus of the vagina that bled very easily on touch. It did appear to be fixed to the underlying structures. Speculum examination was normal, and there was no other abnormality detected. The patient was counseled that in all likelihood this was a benign skin tag. Following consent, the lesion was excised under local anesthetic and sent for histological analysis.

Histology from the vaginal biopsy showed features of an ulcerated and invasive moderately differentiated adenocarcinoma exhibiting endometrioid architecture (Figure 1A). The immunophenotype was positive for cytokeratin (CK) 20 (Figure 1B), carcinoembryonic antigen (CEA) (Figure 1C), and caudal-related homeobox 2 (Figure 1D), with very minor immunoreactivity for CK 7 and negative staining for estrogen receptor, progesterone receptor, vimentin, thyroid transcription factor 1, a primary lung cancer marker, and gross cystic disease fluid protein (GCDFP15), a breast tumor marker.

The histology, although suggestive of a carcinoma of possible endometrial origin, was contradicted by the immunophenotype was highly suggestive of a cancer of colorectal origin and thus the tumor was thought to be of likely metastatic origin.

Tumor markers at the time of presentation were all within normal ranges; CEA $<0.5(<2.5 \mu \mathrm{g} / \mathrm{L})$, Ca 12514 $(<35 \mathrm{kU} / \mathrm{L})$, and $\mathrm{Ca} 19.93(<60 \mathrm{kU} / \mathrm{L})$.
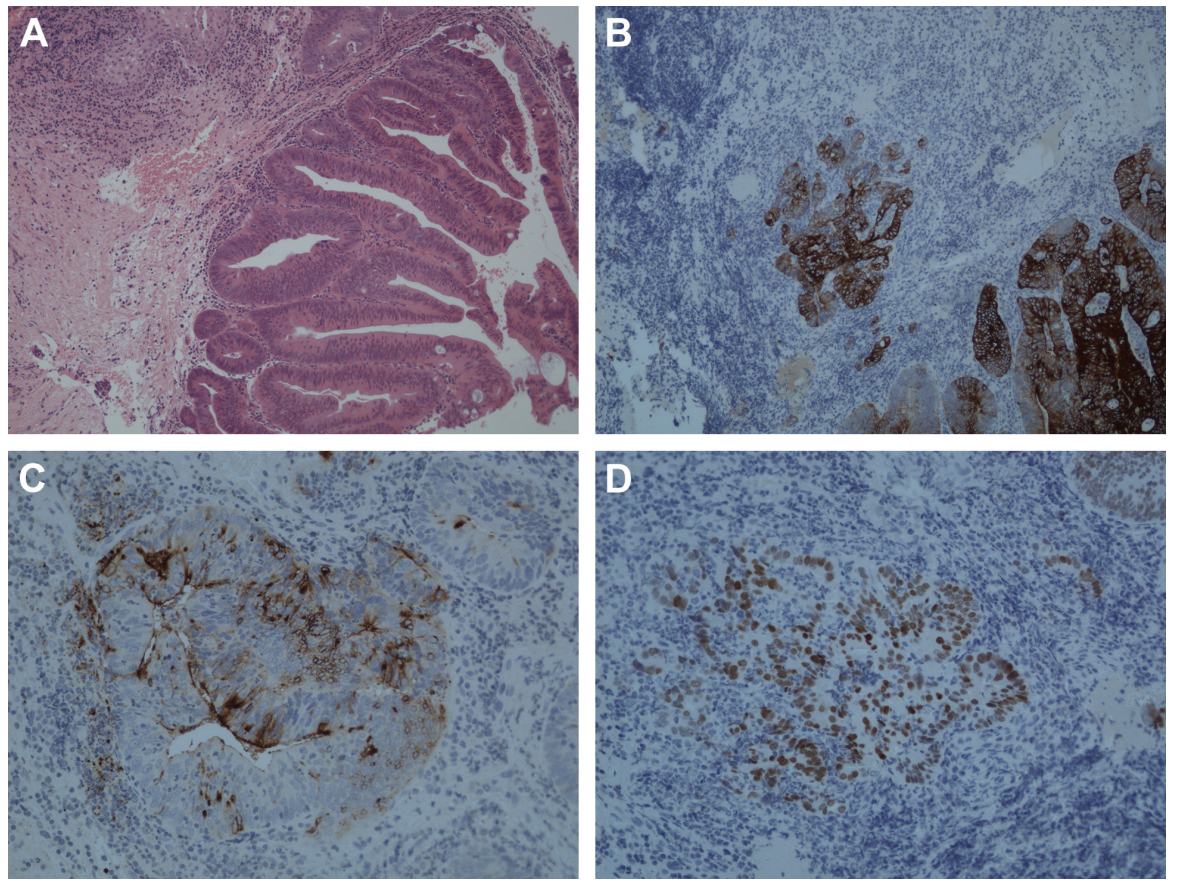

Figure I Immunohistochemistry demonstrated on vaginal biopsy.

Notes: This combination of stains is highly suggestive of a malignancy of gastrointestinal origin. (A) Moderately differentiated adenocarcinoma on H\&E staining at 200X magnification. (B) Positive stain for CK 20 at 200× magnification. (C) Positive stain for CEA at 400× magnification. (D) Positive stain for CDX 2 at $200 \times$ magnification. Abbreviations: CEA, carcinoembryonic antigen; CDX, caudal-related homeobox 2; CK, cytokeratin.

The patient's case was discussed at the gynaecology-oncology multidisciplinary meeting (MDT). As the immunohistochemistry (IHC) analysis suggested that the tumor was of colorectal origin, a referral for gastrointestinal (GI) tract endoscopic screening was made. The patient was brought in for a computerized tomography (CT) chest/abdomen/pelvis. The CT of the abdomen/pelvis demonstrated some non-specific tissue thickening of the perineum/peritoneum, but no evidence of metastatic disease. CT of the chest was normal apart from an isolated non-specific pulmonary nodule which has remained stable on subsequent followup scans and was not characteristic of either a primary or pulmonary metastasis.

On the same admission, she had a wide local excision of the base of the polypoid tumor as a day case under general anesthesia. A $3 \mathrm{~mm}$ by $5 \mathrm{~mm}$ papillomatous lesion of the posterior fourchette was excised with a $1 \mathrm{~cm}$ circumferential margin. The procedure was uncomplicated, and the patient was discharged later that day. Histology showed a focal area of ulceration and granulation tissue which contained a superficial deposit of residual adenocarcinoma, consistent with the same immunophenotype (CK 20 positive/CK 7 negative).

A second opinion was sought from a specialist gynecological pathologist of international repute who confirmed the IHC and suggested that this was a metastasis from a primary tumor and was highly likely to be present somewhere within 
the colorectum, yet to manifest clinically. However, it was suggested to rule out the possibility of a vaginal neoplasm that maybe associated with a rectovaginal fistula.

Upper GI endoscopy proved to be normal and colonoscopy revealed four small polyps. Two $5 \mathrm{~mm}$ sessile polyps in the proximal right colon, one $9 \mathrm{~mm}$ sessile polyp in middle transverse colon, and one $5 \mathrm{~mm}$ polyp in the proximal descending colon were noted and excised. Biopsies of the polyps from the ascending colon and transverse colon showed tubular adenomata with low grade dysplasia. The biopsy from the descending colon polyp showed normal large bowel mucosa only.

With no obvious primary tumor elucidated from endoscopy/ colonoscopy, a proton emission tomography (PET)-CT was organized (Figure 2A-C). This showed an area of increased fluorodeoxyglucose (FDG)-avid uptake to the left of the midline measuring $\sim 2.5 \mathrm{~cm}$ and appeared to be arising from the uterus. Her case was rediscussed in the gynecological oncology MDT. At this stage, it was felt that the FDG-avid area was unlikely to represent an ovary. Although fibroids may explain the increased uptake, a malignant neoplasm could not be excluded in view of the hotspot that was noted (Figure 2C). There was no evidence of uptake in large or small bowel. The lungs were clear and the mediastinum normal. Of note, degenerative changes in the spine particularly involving the facet joints show some increased uptake but no evidence of a destructive lesion was noted. There was no abnormal uptake at area of the original vulval deposit. There was no other evidence suggestive of the site of primary malignancy.

Review at the gynecological MDT concluded that the FDG-avid "hotspot" may indicate an occult primary tumor within the uterus as endometrial tumors do metastasize to the vagina. Therefore, the MDT recommended a hysterectomy along with removal of her tubes and ovaries.
A total laparoscopic hysterectomy, bilateral salpingooophorectomy was performed, and peritoneal washings were sent for cytology. Per-operatively a $3 \mathrm{~cm}$ mass adjacent to the uterus in the left broad ligament was noted. There was no obvious disease on the uterine serosa and cervix, and both ovaries appeared macroscopically normal. There was no nodularity in the omentum. The liver and diaphragm also appeared normal. The procedure was uncomplicated with an estimated blood loss of $100 \mathrm{~mL}$ and following an uneventful post-operative recovery, the patient was discharged the following day. The histology, however, again proved negative with an inactive endometrium, normal cervix and only a benign subserosal leiomyoma in the left lower segment and evidence of post-menopausal atrophy. There was no evidence of hyperplasia, dysplasia or malignancy in any of tissues examined. Peritoneal washings were negative for malignancy.

Following the hysterectomy, the case was again discussed at the MDT. As the histology proved to be negative, a colorectal origin was thought to be most likely and the patient was referred to the cancers of unknown primary clinic. The patient was counseled for genetic testing, however, declined this.

Examination at the oncology clinic confirmed no palpable lymphadenopathy, and breast examination was normal. An initial pelvic magnetic resonance imaging (MRI) and 6 monthly surveillance CT scans for the first 2 years were recommended. As she was asymptomatic with no evidence of residual disease, she was not felt to be a candidate for any adjuvant treatment and hence close surveillance with regular bi-annual colonoscopies was recommended.

MRI was normal apart from low signal change consistent with scarring in the subcutaneous fat of the perineum.

Initially, bi-annual surveillance colonoscopy was performed. It again revealed the following four tiny polyps: one $2 \mathrm{~mm}$ sessile polyp in proximal right colon and three $<2 \mathrm{~mm}$

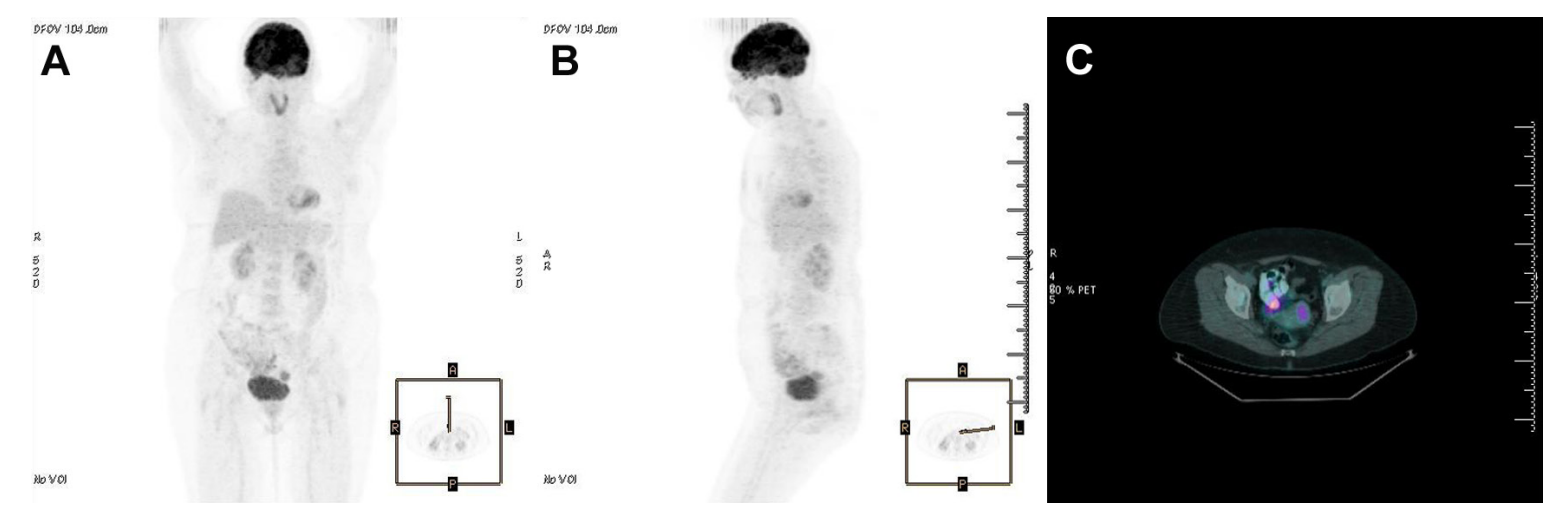

Figure 2 PET-CT demonstrating a uterine FDG-avid "hotspot". (A) Coronal plane. (B) Sagittal plane. (C) Transverse plane. Abbreviations: $\mathrm{CT}$, computerized tomography; PET, proton emission tomography. 
sessile polyps in middle right colon. Histological analysis of all polyps from the cecum and ascending colon showed pieces of sessile tubular adenomata of low grade dysplasia. No invasive adenocarcinoma was seen. Repeat CT showed stable appearances with no identified primary.

As the first two surveillance colonoscopies did not show any disease, follow-up was extended to annual surveillance.

Annual surveillance colonoscopy was normal to the level of the cecum, and the patient remains disease free over 5 years since the initial presentation with ongoing follow-up.

\section{Discussion}

Vaginal skin tags very rarely signify more sinister pathology. Vaginal cancers are rare representing $\sim 1 \%$ of gynecological malignancies and are usually squamous cell carcinomas, associated with oncogenic HPV infection or chronic vaginal irritation, eg, in women with long-standing history of prolapse.

Primary vaginal adenocarcinomas may be endometrioid or clear cell in pattern but with a characteristic immunoprofile as described later with some being associated with pelvic endometriosis.

Colorectal carcinomas are common. They currently represent the third most diagnosed cancer in both men and women, with the third highest mortality rates. ${ }^{1}$

Colonic carcinomas commonly metastasise to the liver and lung. Less frequently they metastasise to other sites including the brain, skeletal muscle and cutaneous tissue. Distant metastases would often indicate disseminated disease.

Vaginal metastases from colorectal cancers are extremely rare, with only a handful of cases being reported in modern literature..$^{2-10}$

When the origin of a new lesion is not immediately apparent, immunohistochemical analysis may be required to aid diagnosis.

Histological analysis can determine cancer classification but cannot discriminate between sites of primary cancers.

The adenomatous polyposis coli (APC) gene has been recognized as a tumor suppressor gene. Mutations in the APC gene have been found in adenomas as well as sporadic cancers. ${ }^{10}$ APC is a multi-domain protein, contains several microtubules and plays a role in the suppression of the Wnt signaling pathway which is responsible for the cell proliferation and differentiation in the intestine. While it may have been valuable for the index case to undergo genetic mutation testing, this was not undertaken as per patient choice.

Imaging techniques including MRI, CT and PET may assist in locating a primary tumor.

Analysis using IHC has revolutionized the identification of metastatic cancers with unknown primaries. It is well accepted that CK profiles are fundamental to this. CKs are intermediate-sized filaments present within the epithelium. Individual CK profiles are of limited use but used in a panel of CKs, they can provide useful information to aid diagnosis by a process of elimination.

The profile of CK 7 negative/CK 20 positive is typical of colorectal primaries, whereas CK 20 negative/CK 7 positive is suggestive of gynecological, breast or lung carcinomas. ${ }^{11-13}$

The pattern of CK 7 negative/CK 20 positive is present in $95 \%$ of colorectal adenocarcinomas. The inverse is true for endometrial cancers where $100 \%$ have the CK 7 positive/ CK 20 negative pattern. ${ }^{12}$

IHC provides invaluable evidence either in collaboration with or in the absence of the previously mentioned diagnostic techniques.

The first presentation in this unusual case was a skin tag with moderately differentiated adenocarcinoma but with a surprising CK 20 positive/CK 7 negative immunophenotype, highly suggestive of a bowel primary. ${ }^{1-13}$ This probable diagnosis is supported by the patient's strong family history of GI malignancies. While we performed a hysterectomy and removal of both tubes and ovaries in the hunt for an occult primary lesion, in fact this proved to be unnecessary and perhaps she could have been managed more conservatively with surveillance only. Certainly for a younger woman in her reproductive years, close surveillance with repeat hysteroscopies and endometrial sampling may have been a reasonable alternative.

Only a handful of colorectal cancers metastasising to the vagina have been reported in modern literature. This case is of further intrigue as although the suspected primary site is thought to be of colorectal origin, no primary tumor has yet been identified, making this a cancer of unknown origin.

Cancers of unknown primary origin (CUP) account for $3 \%-5 \%$ of all cancers, and prognosis overall can be very poor. ${ }^{14}$ The median survival of all CUPs is as low as $4-12$ months. However, this is inclusive of all CUPs, in reality there is great differentiation of outcomes between different histological subtypes and isolated or widespread metastatic lesions. Adenocarcinomas are the most frequently diagnosed. The next step is to use immunohistochemical markers help to differentiate sites of origin. Immunophenotype is thought to be a reliable indicator of origin site, although evidence pertaining to the accuracy of immunohistochemical staining is all from samples in which the primary is known. Colonic cancer CUP has the most favorable outcome.

The diagnostic algorithm from Stella et a ${ }^{14}$ allowing for the differentiation of adenocarcinomas of unknown primaries 
supports our theory that this vaginal skin tag of adenocarcinoma is very likely to be of colonic origin.

However, there is little evidence that determining the origin has any bearing on outcome. ${ }^{14}$

New generation technology to identify tissue of origin (toO) such as microgene assays using PCR is being developed. ${ }^{15}$ Molecular profiling for the identification of toO is a promising new technique. Early clinical trials are underway. ${ }^{16,17}$

The label of CUP has become a diagnosis in its own right. Therefore, treatment recommendations have been devised. The diagnosis of CUP must first be divided into favorable or unfavorable outcomes. Individualized management is favorable in CUPs, with MDT approach between radiologists, biochemists and oncologists. In this particular case, there was an isolated metastasis not extensive multiple metastases, therefore there would be limited benefit gained by systemic treatments.

According to ESMO clinical guidance, this patient would fall into the subset of single metastatic deposit in which the recommendation is resection \pm radiotherapy, \pm systemic treatment. Our patient had a single metastatic deposit which was resected, and the basis of the decision to withhold systemic treatments was made because the patient was asymptomatic with no evidence of recurrence 2 years post diagnosis. ${ }^{18}$ It is noted that there is no indication for routine follow-up for asymptomatic patients, although specific investigations may be indicated. In this case, regular surveillance colonoscopies are indicated.

Although no primary has yet been identified, it is still thought that an indolent bowel primary is the likely diagnosis. As the patient is asymptomatic with no clinical or radiological evidence for recurrence, no treatment has been offered. As cancers of unknown primaries generally have poor long-term survival, regular surveillance is essential to rule out future recurrence.

In summary, this unusual case of a vaginal skin tag revealed an adenocarcinoma with endometrioid architecture and found to have an immunophenotype suggestive of colorectal origin. Colonic cancers can rarely metastasise to the vagina, and such cases usually represent extensive disseminated disease. This case is interesting, as the vaginal lesion appears to be the first presentation of an occult colorectal cancer. Further intrigue arises as despite extensive investigations, no primary malignancy has yet been identified.

\section{Consent for publication}

Written informed consent for publication of the case details and accompanying images was obtained from the patient prior to preparing the manuscript.

\section{Acknowledgment}

Our sincere thanks to Mr Simon Butler-Manuel (consultant gynecological oncologist) for his assistance in proofreading the manuscript.

\section{Disclosure}

The authors report no conflicts of interest in this work.

\section{References}

1. Siegel R, Naishadham D, Jemal A. Cancer statistics, 2012. CA Cancer J Clin. 2012;62(1):10-29.

2. Tanaka T, Kanda T, Sakaguchi S, Munakata S, Ohmichi M. Vaginal stump metastasis from sigmoid colon cancer. Acta Cytol. 2012;56(1): 92-96.

3. Marchal F, Leroux A, Hoffstetter S, Granger P. Vaginal metastasis revealing colon adenocarcinoma. Int $J$ Colorectal Dis. 2006;21(8) $861-862$.

4. Ceccaroni M, Paglia A, Ruffo G, et al. Symptomatic vaginal bleeding in a postmenopausal woman revealing colon adenocarcinoma metastasizing exclusively to the vagina. J Minim Invasive Gynecol. 2010;17(6): 779-781.

5. Chagpar A, Kanthan SC. Vaginal metastasis of colon cancer. Am Surg. 2001;67(2):171-172.

6. Lorente L, Alonso S, Pascual M, Pera M. Vaginal metastasis of colon cancer. Rev Esp Enferm Dig. 2011;103(8):435-436.

7. Sabbagh C, Fuks D, Regimbeau JM, Degremont R, Jarry-Tossou V, Mauvais F. Isolated vaginal metastasis from rectal adenocarcinoma: a rare presentation. Colorectal Dis. 2011;13(10):e355-e356.

8. Funada T, Fujita S. A case of vaginal metastasis from a rectal cancer. Jpn J Clin Oncol. 2010;40(5):482.

9. Katsumoto Y, Maruyama K, Furukawa J, et al. [A case of metastatic vaginal tumor of rectal cancer]. Gan To Kagaku Ryoho. 2002;29(12): 2406-2409. Japanese.

10. Lee SM, Whiteley HW. Unusual metastatic sites of colonic and rectal carcinoma: report of four cases. Dis Colon Rectum. 1974;17(4):560-561.

11. Bayrak R, Haltas H, Yenidunya S. The value of CDX2 and cytokeratins 7 and 20 expression in differentiating colorectal adenocarcinomas from extraintestinal gastrointestinal adenocarcinomas: cytokeratin 7-/20+ phenotype is more specific than CDX2 antibody. Diagn Pathol. 2012;23:7-9.

12. Chu P, Wu E, Weiss LM. Cytokeratin 7 and cytokeratin 20 expression in epithelial neoplasms: a survey of 435 cases. Mod Pathol. 2000; 13(9):962-972.

13. Tot T. Cytokeratins 20 and 7 as biomarkers: usefulness in discriminating primary from metastatic adenocarcinoma. Eur J Cancer. 2002;38(6): 758-763.

14. Stella GM, Senetta R, Ronco M, Cassoni P. Cancers of unknown primary origin: current perspectives and future therapeutic strategies. J Transl Med. 2012;24:10-12.

15. Greco FA, Spigel DR, Yardley DA, Erlander MG, Ma XJ, Hainsworth JD Molecular profiling in unknown primary cancer: accuracy of tissue of origin prediction. Oncologist. 2010;15(5):500-506.

16. Review of molecular profiling-real time polymerase chain reaction (RT-PCR) in unknown primary cancer. NCT00876408. Available from: www.clinicaltrials.gov. Accessed February 20, 2018.

17. Treatment based on molecular profiling diagnosis carcinoma of unknown primary site. NCT00737243. Available from: www.clinicaltrials.gov. Accessed February 20, 2018.

18. Fizazi K, Greco FA, Pavlidis N, et al. Cancers of unknown primary site: ESMO clinical practice guidelines for diagnosis, treatment and follow-up. Ann Oncol. 2015;26(Suppl 5):v133-v138. 


\section{Publish your work in this journal}

The International Journal of Women's Health is an international, peerreviewed open-access journal publishing original research, reports, editorials, reviews and commentaries on all aspects of women's healthcare including gynecology, obstetrics, and breast cancer. The manuscript management system is completely online and includes

a very quick and fair peer-review system, which is all easy to use. Visit http://www.dovepress.com/testimonials.php to read real quotes from published authors.

Submit your manuscript here: http://www.dovepress.com/international-journal-of-womens-health-journal 